\title{
Compactly Supported Tight and Sibling Frames Based on Generalized Bernstein Polynomials
}

\author{
Ting Cheng and Xiaoyuan Yang \\ Department of Mathematics, Beihang University, LMIB of the Ministry of Education, Beijing 100191, China \\ Correspondence should be addressed to Xiaoyuan Yang; xiaoyuanyang@vip.163.com
}

Received 30 December 2015; Revised 28 April 2016; Accepted 3 May 2016

Academic Editor: Raffaele Solimene

Copyright ( 92016 T. Cheng and X. Yang. This is an open access article distributed under the Creative Commons Attribution License, which permits unrestricted use, distribution, and reproduction in any medium, provided the original work is properly cited.

\begin{abstract}
We obtain a family of refinable functions based on generalized Bernstein polynomials to provide derived properties. The convergence of cascade algorithms associated with the new masks is proved, which guarantees the existence of refinable functions. Then, we analyze the symmetry, regularity, and approximation order of the refinable functions, which are of importance. Tight and sibling frames are constructed and interorthogonality of sibling frames is demonstrated. Finally, we give numerical examples to explicitly illustrate the construction of the proposed approach.
\end{abstract}

\section{Introduction}

Because it is highly desirable to construct wavelets within a class of analytically representable functions, compactly supported sibling frames with interorthogonality attract a considerable amount of attention, recently.

In 1997, Ron and Shen completed the structure of the affine system, which can be factored during a multiresolution analysis construction. This leads to a characterization of all tight frames that can be constructed by the methods in [1]. In 2000, compactly supported tight frames that correspond to refinable functions were studied and a constructive proof was given by Chui and He [2]. In [3], Han gave his investigation of symmetric tight framelet filter banks with a minimum number of generators and systematically studied them with three high-pass filters which are derived from the oblique extension principle. In 2002, compactly supported tight and sibling frames, with symmetry (or antisymmetry), minimum support, shift-invariance, and interorthogonality, were constructed in [4]. In 2003, Daubechies et al. discussed wavelet frames constructed via multiresolution analysis, with emphasis on tight wavelet frames. More importantly, they established general principles and specific algorithms for constructing framelets and tight framelets in [5]. In 2005, Averbuch et al. [6] obtained tight and sibling frames originated from discrete splines, in which, all the filters are linear phase and generate symmetric scaling functions with analysis and synthesis pairs of framelets. Next, in [7], symmetric wavelets dyadic sibling and dual frames, where each of the frames consists of three generators obtained using spectral factorization, were given. In 2007, a new type of pseudo-splines was introduced to construct symmetric or antisymmetric tight framelets with desired approximation orders by Dong and Shen [8]. And they provided various constructions of wavelets and framelets. In 2013, Shen and $\mathrm{Xu}$ [9] give $B$-Spline framelets derived from the unitary extension principle, which led to the result that the wavelet system is generated by finitely many consecutive derivatives. More tight frames have been studied in [10-20], so far.

This paper is concerned with the construction of compactly supported tight and sibling frames based on generalized Bernstein polynomials [21], defined as

$$
S_{k}^{(n)}(t)=\left(\begin{array}{l}
n \\
k
\end{array}\right) \frac{t(t+\alpha) \cdots(t+[k-1] \alpha)(1-t)(1-t+\alpha) \cdots(1-t+[n-k-1] \alpha)}{(1+\alpha)(1+2 \alpha) \cdots(1+[n-1] \alpha)}
$$


where $\alpha \geq 0$. We complete the convergence of cascade algorithms associated with the new masks. Furthermore, the symmetry, regularity, and approximation orders of corresponding refinable functions are analyzed. At last, we implement interorthogonality of sibling frames.

The remainder of this paper is organized as follows. In Section 2, some notations about refinement marks are collected and some technical lemmata are given to use in other sections. We will elaborate on convergence of cascade algorithms based on the masks, which guarantees the existence of refinable functions in Section 3. Section 4 analyzes the symmetry and gives a symmetry proof. In Section 5, regularity and approximation orders are focused on study; at the same time, we obtain the lower bound of the regularity exponents of refinable functions by estimating the decay rates of their Fourier transform. At last, we construct tight and sibling frames and obtain interorthogonality of sibling frames in Section 6.

\section{Preliminaries}

For the convenience of the readers, we review some definitions and properties about refinement marks in this section.

New marks based on generalized Bernstein polynomials (1), with order $(m, l, \alpha)$, for given nonnegative integers $m, l$, and $\alpha \geq 0$, are defined as follows:

$$
\tau^{m, l, \alpha}(\omega):=\cos ^{2}\left(\frac{\omega}{2}\right)\left(\cos ^{2}\left(\frac{\omega}{4}\right)-\frac{\sum_{j=0}^{l}\left(\begin{array}{c}
m+l \\
j
\end{array}\right)\left(\prod_{k=0}^{j-1}\left(\cos ^{2}(\omega / 2)+k \alpha\right) \prod_{k=0}^{m+l-j-1}\left(\sin ^{2}(\omega / 2)+k \alpha\right)\right)}{\prod_{k=1}^{m+l-1}(1+k \alpha)}\right) .
$$

For notational simplicity, we will introduce the following two definitions:

$$
\begin{aligned}
B_{j}^{m, l, \alpha}(\omega) & :=\frac{\left(\prod_{k=0}^{j-1}\left(\cos ^{2}(\omega / 2)+k \alpha\right) \prod_{k=1}^{m+l-j-1}\left(\sin ^{2}(\omega / 2)+k \alpha\right)\right)}{\prod_{k=1}^{m+l-1}(1+k \alpha)}, \\
T^{m, l, \alpha}(\omega): & \cos ^{2}\left(\frac{\omega}{4}\right)-\frac{\sum_{j=0}^{l}\left(\begin{array}{c}
m+l \\
j
\end{array}\right)\left(\prod_{k=0}^{j-1}\left(\cos ^{2}(\omega / 2)+k \alpha\right) \prod_{k=1}^{m+l-j-1}\left(\sin ^{2}(\omega / 2)+k \alpha\right)\right)}{\prod_{k=1}^{m+l-1}(1+k \alpha)} .
\end{aligned}
$$

By $L_{2}(\mathbb{R})$, we denote all the functions $f(x)$ satisfying

$$
\|f(x)\|_{L_{2}(\mathbb{R})}:=\left(\int_{\mathbb{R}}|f(x)|^{2} d x\right)^{1 / 2}<\infty
$$

and $l_{2}(\mathbb{Z})$ the set of all sequences $c$ defined on $\mathbb{Z}$ such that

$$
\|c\|_{l_{2}(\mathbb{Z})}:=\left(\sum_{k \in \mathbb{Z}}|c(k)|^{2}\right)^{1 / 2}<\infty
$$

In the following, we will give a compactly supported realvalued refinable function $\phi: \mathbb{R} \rightarrow \mathbb{R}$ with finite mask and real mask coefficients; that is, $\phi$ satisfies a two-scale relation:

$$
\phi(x)=\sum_{k=N_{1}}^{k=N_{2}} p_{k} \phi(2 x-k), \quad \text { a.e. } x \in \mathbb{R}
$$

for some real numbers $p_{k}$. Assume that the corresponding two-scale Laurent polynomials

$$
P(z):=\frac{1}{2} \sum_{k=N_{1}}^{k=N_{2}} p_{k} z^{k}
$$

satisfy

$$
P(z)=\left(\frac{1+z}{2}\right)^{m} P_{0}(z)
$$

for some $m \geq 1$, with a Laurent polynomial $P_{0}$ that satisfies $P_{0}(-1) \neq 0$.

The Fourier transform of $\phi$ is

$$
\widehat{\phi}(\xi)=\int_{\mathbb{R}} \phi(t) e^{-i \xi t} d t, \quad \xi \in \mathbb{R}
$$

And, $\phi$ satisfies

$$
\widehat{\phi}(0)=1 \text {. }
$$

With the above, the refinement equation (6) can be written in terms of its Fourier transform as

$$
\widehat{\phi}(\xi)=\tau\left(\frac{\xi}{2}\right) \widehat{\phi}\left(\frac{\xi}{2}\right), \quad \xi \in \mathbb{R}
$$

where $\tau(\xi / 2)=P(z), z=e^{-i \xi / 2}$. We call $\tau$ the refinement mask for convenience, too.

By the iteration of (11), the corresponding refinable function $\phi$ can be written in terms of its Fourier transform as

$$
\widehat{\phi}(\omega):=\prod_{j=1}^{\infty} \tau\left(2^{-j} \omega\right) .
$$


In the following, we will adopt some of the notations from $[2,4,22]$. The transition operator $T_{\widehat{a}}$ for $2 \pi$-periodic functions $\widehat{a}$ and $f$ can be defined as

$$
\begin{aligned}
{\left[T_{\widehat{a}} f\right](w):=} & \left|\widehat{a}\left(\frac{\omega}{2}\right)\right|^{2} f\left(\frac{\omega}{2}\right) \\
& +\left|\widehat{a}\left(\frac{\omega}{2}+\pi\right)\right|^{2} f\left(\frac{\omega}{2}+\pi\right), \quad \omega \in \mathbb{R} .
\end{aligned}
$$

For $\tau \in \mathbb{R}$, a quantity is defined by

$$
\rho_{\tau}(\widehat{a}, \infty):=\limsup _{n \rightarrow \infty}\left\|T_{\widehat{a}}^{n}\left(\left|\sin \left(\frac{\omega}{2}\right)\right|^{\tau}\right)\right\|_{L_{\infty}(\mathbb{I})}^{1 / n} .
$$

The notation $\rho(\widehat{a})$ is defined by

$$
\begin{aligned}
& \rho(\widehat{a}):=\inf \left\{\rho_{\tau}(\widehat{a}, \infty):|\widehat{a}(\omega+\pi)|^{2}\left|\sin \left(\frac{\omega}{2}\right)\right|^{\tau}\right. \\
& \left.\quad \in L_{\infty}(\mathbb{T}), \tau \geq 0\right\} .
\end{aligned}
$$

For convenience, assume that

$$
\phi \text { is piecewise } \operatorname{Lip} \alpha, \quad \text { for some } \alpha>0 \text {. }
$$

The family $\Psi=\left\{\psi_{1}, \ldots, \psi_{n}\right\} \subset L^{2}$ is interorthogonal if $W^{i} \perp W^{j}, i \neq j$, where $W^{i}=\operatorname{clos}_{L^{2}} \operatorname{span}\left\{\psi_{i}(x-k): k \in \mathbb{Z}\right\}$.

The modulus of continuity of a function $f$ defined on an open interval $I=(c, d)$ will be denoted, as usual, by

$$
\omega(f, I ; t):=\sup _{\substack{c<x_{1}<x_{2}<d \\\left|x_{2}-x_{1}\right|<t}}\left|f\left(x_{2}\right)-f\left(x_{1}\right)\right| .
$$

A function $f$ defined on the real line $\mathbb{R}$ is called a piecewise Lip $\alpha$ function, $\alpha>0$, if there exist finitely many values $-\infty<$ $t_{1}<\cdots<t_{s}<\infty$, such that

$$
\omega\left(f, I_{j} ; t\right)=O\left(t^{\alpha}\right), \quad j=0, \ldots, s,
$$

where $I_{0}:=\left(-\infty, t_{1}\right), I_{1}:=\left(t_{1}, t_{2}\right), \ldots, I_{s-1}:=\left(t_{s-1}, t_{s}, I_{s}:=\right.$ $\left.\left(t_{s}, \infty\right)\right)$.

Two finite families, $\left\{\psi_{i}\right\},\left\{\widetilde{\psi}_{i}\right\} \in L^{2}$, are defined by scaling relations

$$
\begin{aligned}
& \widehat{\psi}_{i}(\omega)=Q_{i}(z) \hat{\phi}\left(\frac{\omega}{2}\right) \\
& \widehat{\widetilde{\psi}}_{i}(\omega)=\widetilde{Q}_{i}(z) \hat{\phi}\left(\frac{\omega}{2}\right), \\
& i=1, \ldots, N,
\end{aligned}
$$

where $Q_{i}(z), \widetilde{Q}_{i}(z)$ are Laurent polynomials that have real coefficients and vanish at $z=1$. In other words,

$$
\begin{aligned}
& Q_{i}(z)=\left(\frac{1-z}{2}\right)^{m_{i}} q_{i}(z), \\
& \widetilde{Q}_{i}(z)=\left(\frac{1-z}{2}\right)^{\widetilde{m_{i}}} \widetilde{q_{i}}(z),
\end{aligned}
$$

where $m_{i}, \widetilde{m}_{i} \geq 1$. Hence, the functions $\psi_{i}$ and $\widetilde{\psi}_{i}$ have compact support and at least one vanishing moment.

A function $f$ belongs to the Hölder class $C^{\beta}(\mathbb{T})$ with $\beta>$ 0 , if $f$ is a $2 \pi$-periodic continuous function such that $f$ is $n$ times continuously differentiable and there exists a positive number $C$ satisfying

$$
\left|f^{(n)}(x)-f^{(n)}(y)\right| \leq C|x-y|^{\beta-n}
$$

for all $x, y \in \mathbb{T}$, where $n$ is the largest integer such that $n \leq \beta$.

We use

$$
P_{n}: f \longmapsto \sum_{k \in \mathbb{Z}}\left\langle f, \phi_{n, k}\right\rangle \phi_{n, k}
$$

for approximation of $f \in L_{2}(\mathbb{R})$. And a function $\phi$ satisfies the Strang-Fix condition of order $m$ if

$$
\begin{aligned}
\widehat{\phi}(0) & \neq 0, \\
D^{j} \widehat{\phi}(2 \pi k) & =0,
\end{aligned}
$$

$$
\forall k \in \mathbb{Z} \backslash\{0\}, \forall|j|<m .
$$

Under certain conditions on $\phi$ (e.g., if it is compactly supported and $\widehat{\phi}(0)=1$ ), the Strang-Fix condition is equal to the requirement that $\widehat{\tau_{0}}$ has a zero of order $m$ at each of the points in $\{0, \pi\} \backslash 0$. In [5], if $\phi$ satisfies the Strang-Fix condition of order $m$ and the corresponding mark $\tau$ satisfies that $1-|\tau(\cdot)|^{2}=O\left(|\cdot|^{m_{1}}\right)$ at the origin, then the approximation order is $\min \left\{m, m_{1}\right\}$.

We will provide some lemmas which are necessary for the following theorem. The following lemmas are about the relations of the quantities $\rho_{\tau}(\hat{a}, \infty)$ associated with masks and a condition of the convergence of cascade algorithms.

Lemma 1 (see [22, Theorem 4.1]). Let $\hat{a}$ be a $2 \pi$-periodic measurable function such that $|\widehat{a}|^{2} \in C^{\beta}(\mathbb{T})$ with $|\widehat{a}|^{2}(0) \neq 0$ and $\beta>0$. If $|\widehat{a}(\omega)|^{2}=\left|1+e^{-i \omega}\right|^{2 \tau}|\widehat{A}(\omega)|^{2}$ a.e. $\omega \in \mathbb{R}$ for some $\tau \geq 0$ such that $\widehat{A}(\omega) \in L_{\infty}(\mathbb{T})$, then

$$
\begin{aligned}
\rho_{2 \tau}(\widehat{a}, \infty) & =\inf _{n \in \mathbb{N}}\left\|T_{\widehat{a}}^{n} 1\right\|_{L_{\infty}(\mathbb{T})}^{1 / n}=\lim _{n \rightarrow \infty}\left\|T_{\widehat{a}}^{n} 1\right\|_{L_{\infty}(\mathbb{T})}^{1 / n} \\
& =\rho_{0}(\widehat{A}, \infty) .
\end{aligned}
$$

Lemma 2 (see [22, Theorem 4.3]). Let $\widehat{a}$ and $\widehat{c}$ be $2 \pi$-periodic measurable functions such that

$$
|\widehat{a}(\omega)| \leq|\widehat{c}(\omega)|
$$

for almost every $\omega \in \mathbb{R}$. Then

$$
\rho_{\tau}(\widehat{a}, \infty) \leq \rho_{\tau}(\widehat{c}, \infty), \quad \tau \in \mathbb{R}
$$

Lemma 3 (see [22, Theorem 2.1]). Let $\widehat{a} \in C^{\beta}(\mathbb{T})$ with $\widehat{a}(0)=$ 1 and $\beta>0$. If $\rho(\widehat{a})<1$, then the cascade algorithm associated with the mask $\widehat{a}$ converges in the space $L_{2, \infty}(\mathbb{R})$.

For regularity, our primary goal is to obtain the lower bound of its exponents $\gamma_{0}^{m, l, \alpha}$ of refinable functions $\phi^{m, l, \alpha}$ by 
estimating the decay rates $\beta_{0}^{m, l, \alpha}$ of their Fourier transform. The relation is expressed by

$$
\gamma_{0}^{m, l, \alpha} \geq \beta_{0}^{m, l, \alpha}-1-\varepsilon,
$$

for any small enough $\varepsilon>0$; see [23]. Consequently, $\phi^{m, l, \alpha} \in$ $C^{\gamma_{0}^{m l, \alpha}}$. Next, we will give an estimate of the decay rates $\beta_{0}^{m, l, \alpha}$ of the Fourier transform of refinable functions $\phi^{m, l, \alpha}$ with the mask $\tau^{m, l, \alpha}(\omega)$. By $[23,24]$, for any stable, compactly supported refinable functions $\phi$ in $L_{2}(\mathbb{R})$ with $\widehat{\phi}(0)=1$, the refinement mask $\tau$ must satisfy $\tau(0)=1$ and $\tau(\pi)=0$. Thus, $\tau$ can be factorized as

$$
\tau(\omega)=\cos ^{n}\left(\frac{\omega}{2}\right) \mathscr{L}(\omega)
$$

where $n$ is the maximal multiplicity of the zeros of $\tau$ at $\pi$ and $\mathscr{L}(\omega)$ is a trigonometric polynomial with $\mathscr{L}(0)=1$. Therefore, one obtains

$$
\begin{aligned}
\widehat{\phi}(\omega) & =\prod_{j=1}^{\infty} \tau\left(2^{-j} \omega\right)=\prod_{j=1}^{\infty} \cos ^{n}\left(2^{-j} \frac{\omega}{2}\right) \prod_{j=1}^{\infty} \mathscr{L}\left(2^{-j} \omega\right) \\
& =\operatorname{sinc} c^{n}\left(\frac{\omega}{2}\right) \prod_{j=1}^{\infty} \mathscr{L}\left(2^{-j} \omega\right),
\end{aligned}
$$

which shows the decay of $|\phi|$ can be characterized by $|\tau|$ as stated in the following lemma.

Lemma 4. Let $\tau$ be the refinement mask of the refinable function $\phi$ of the form

$$
|\tau(\omega)|=\cos ^{n}\left(\frac{\omega}{2}\right)|\mathscr{L}(\omega)|, \quad \omega \in[-\pi, \pi] .
$$

If

$$
\begin{gathered}
|\mathscr{L}(\omega)| \leq\left|\mathscr{L}\left(\frac{2 \pi}{3}\right)\right| \quad \text { for }|\omega| \leq \frac{2 \pi}{3}, \\
|\mathscr{L}(\omega) \mathscr{L}(2 \omega)| \leq\left|\mathscr{L}\left(\frac{2 \pi}{3}\right)\right|^{2} \quad \text { for } \frac{2 \pi}{3} \leq|\omega| \leq \pi,
\end{gathered}
$$

then $\widehat{\phi}(\omega) \leq C(1+|\omega|)^{-n+\mathscr{K}}$ with $\mathscr{K}=\log (|\mathscr{L}(2 \pi / 3)|) / \log 2$, and this decay is optimal.

The following lemmas are useful for obtaining the important tight and sibling frames.

Lemma 5 (see [4, Theorem 2]). For any compactly supported refinable function $\phi$ that satisfies (8)-(16), there exist compactly supported sibling frames $\left\{\psi_{1}, \psi_{2}\right\},\left\{\widetilde{\psi}_{1}, \widetilde{\psi}_{2}\right\}$ with the property that all of the four functions have $m$ vanishing moments, where $m$ is the order of the root $z=-1$ of the two-scale Laurent polynomial P. Furthermore, if $\phi$ is symmetric, then all of the four functions can be chosen to be symmetric for even $m$ and antisymmetric for odd $m$.

Lemma 6 (see [4, Theorem 3]). For any compactly supported refinable function $\phi$ that satisfies (8)-(16), there exists a pair of sibling frames $\left(\psi_{1}, \psi_{2}\right)$ and $\left(\widetilde{\psi}_{1}, \widetilde{\psi}_{2}\right)$ such that all of the four functions have compact support and the maximum number $m$ of vanishing moments and that $\left(\psi_{1}, \psi_{2}\right)$ is interorthogonal.
Lemma 7 (see [4, Theorem 8]). Let $\{\psi\},\{\widetilde{\psi}\}$ be a pair of compactly supported sibling frames associated with a $V M R$ function S. If $S$ is Laurent polynomial, then the function $\psi_{t} \in V_{1}$ with two-scale symbol $Q_{t}$, where $Q_{t}=$ $(z / \sqrt{S(-1)}) S(z) P(-1 / z)$, defines a tight frame of $L^{2}$ which is associated with the same VMR function $S$.

\section{Convergence of Cascade Algorithms Based on the Masks}

In this section, demonstration of the convergence of cascade algorithms in the space $L_{2, \infty}(\mathbb{R})$ is given. To complete it, a useful condition of proving the convergence of cascade algorithms is described as follows.

Lemma 8. For two positive integers $l, m, l<m-5$, if

$$
0 \leq \alpha<\frac{1}{3(m+l)-7}
$$

then

$$
\max _{\omega \in \mathbb{T}} B_{j}(\omega) \leq\left(\frac{1}{2}\right)^{m+l-1}, \quad j=1,2, \ldots, l .
$$

Proof. For $j=1,2, \ldots, l$, it is obvious that

$$
B_{j}(\omega)=\frac{\sin ^{2}(\omega / 2)+(m+l-1-j) \alpha}{\cos ^{2}(\omega / 2)+j \alpha} B_{j+1}(\omega) .
$$

We claim that

$$
\frac{B_{j}(\omega)}{B_{j+1}(\omega)}=\frac{\sin ^{2}(\omega / 2)+(m+l-1-j) \alpha}{\cos ^{2}(\omega / 2)+j \alpha}>1 .
$$

Since $l<m-5$, for $j=1,2, \ldots, l$, it holds that

$$
j<m+l-1-j .
$$

There are two cases to consider.

Case 1. Suppose that $\sin (\omega) \geq 0$. By (32) and (36), it is easy to see that

$$
\alpha>0>\frac{-\sin (\omega)}{m+l-1-2 j} .
$$

Then

$$
\sin ^{2}\left(\frac{\omega}{2}\right)+(m+l-1-j) \alpha>\cos ^{2}\left(\frac{\omega}{2}\right)+j \alpha .
$$

This implies condition (35).

Case 2. Suppose that $\sin (\omega)<0$. In the same way, we get

$$
\alpha>\frac{-\sin (\omega)}{m+1-1-2 j},
$$

for $j=1,2, \ldots, l$. Then (38) holds. This concludes claim (35). By using (32), one gets

$$
\begin{aligned}
& (4(m+l-2)-(m+l-1)) \alpha \\
& \quad<\frac{4(m+l-2)-(m+l-1)}{3(m+l)-7}=1 .
\end{aligned}
$$


Then

$$
\frac{(m+l-2) \alpha}{(1+\alpha)(1+(m+l-1) \alpha)}<\frac{(m+l-2) \alpha}{1+(m+l-1) \alpha}<\frac{1}{4} .
$$

Thus,

$$
\begin{aligned}
& (2(m+l-3)-(m+l-2)) \alpha \\
& \quad<\frac{2(m+l-3)-(m+l-2)}{m+l-4}=1 .
\end{aligned}
$$

Similarly, one has

$$
\frac{(m+l-3) \alpha}{1+(m+l-2) \alpha}<\frac{1}{2}
$$

For any $x$, notice that

$$
\left(\frac{x}{1+(1+x)}\right)^{\prime}>0
$$

and $B_{1}(\omega)$, which is a continuous function on $[-\pi, \pi]$ and is differentiable on $(-\pi, \pi)$, has the maximum value at $\omega=\pi$. The reason is as follows: the equation $\left[B_{1}(\omega)\right]^{\prime}=0$ has three zeros, at $\omega=0, \pm \pi$. Since $\left[B_{1}(\omega)\right]^{\prime \prime}>0, B_{1}(0)$ is the minimum of $B_{1}(\omega)$ on $[-\pi, \pi]$. Thus, $B_{1}( \pm \pi)$ is the maximum of $B_{1}(\omega)$ on $[-\pi, \pi]$. Therefore, applying (35), (41), (43), (44), and

$$
\begin{aligned}
B_{1}(\omega)= & \frac{\left(\cos ^{2}(\omega / 2) \prod_{k=1}^{m+l-2}\left(\sin ^{2}(\omega / 2)+k \alpha\right)\right)}{\prod_{k=1}^{m+l-1}(1+k \alpha)} \\
\leq & \frac{\prod_{k=1}^{m+l-2} k \alpha}{\prod_{k=1}^{m+l-1}(1+k \alpha)} \\
\leq & \prod_{k=1}^{m+l-3} \frac{k \alpha}{1+(k+1) \alpha} \\
& \cdot \frac{(m+l-2) \alpha}{(1+\alpha)(1+(m+l-1) \alpha)} \\
\leq & \left(\frac{(m+l-3) \alpha}{1+(m+l-2) \alpha}\right)^{m+l-3} \cdot \frac{1}{4}=\left(\frac{1}{2}\right)^{m+l-1},
\end{aligned}
$$

we get inequality (33).

Theorem 9. For two positive integers $l, m$, satisfying $l<m-5$, if we let $\tau^{m, l, \alpha}(\omega)$ be mask (2), then the cascade algorithm associated with the mask $\tau^{m, l, \alpha}(\omega)$ converges in the space $L_{2, \infty}(\mathbb{R})$.

Proof. For $|\cos (\omega / 2)|=\left|\left(1+e^{-i \omega}\right) / 2\right|$, one has

$$
\begin{aligned}
\left|\tau^{m, l, \alpha}(\omega)\right|^{2} & =2^{-4}\left(1+e^{-i \omega}\right)^{4}|T(\omega)|^{2} \\
& =\left(1+e^{-i \omega}\right)^{4}\left|2^{-2} T(\omega)\right|^{2} .
\end{aligned}
$$

Applying

$$
\begin{aligned}
B_{0}(\omega) & =\frac{\prod_{k=0}^{m+l-1}\left(\sin ^{2}(\omega / 2)+k \alpha\right)}{\prod_{k=1}^{m+l-1}(1+k \alpha)} \\
& \leq \frac{\prod_{k=1}^{m+l-1}(1+k \alpha)}{\prod_{k=1}^{m+l-1}(1+k \alpha)}=1
\end{aligned}
$$

and Lemma 8, we obtain

$$
\begin{aligned}
& \max _{\omega \in \mathbb{T}} 2^{-4}\left|T^{m, l, \alpha}(\omega)\right|^{2}=\left.\max _{\omega \in \mathbb{T}} 2^{-4}|| \cos ^{2}\left(\frac{\omega}{4}\right)\right|^{2} \\
& +\left|\sum_{j=0}^{l}\left(\begin{array}{c}
m+l \\
j
\end{array}\right) B_{j}(\omega)\right|^{2} \\
& -2\left|\cos ^{2}\left(\frac{\omega}{4}\right)\right|\left|\sum_{j=0}^{l}\left(\begin{array}{c}
m+l \\
j
\end{array}\right) B_{j}(\omega)\right| \mid \\
& =\left.\max _{\omega \in \mathbb{T}} 2^{-4}|| \cos ^{2}\left(\frac{\omega}{4}\right)\right|^{2} \\
& +\left|B_{0}(\omega)+\sum_{j=1}^{l}\left(\begin{array}{c}
m+l \\
j
\end{array}\right) B_{j}(\omega)\right|^{2} \\
& -2\left|\cos ^{2}\left(\frac{\omega}{4}\right)\right| \mid B_{0}(\omega)+\sum_{j=1}^{l}\left(\begin{array}{c}
m+l \\
j
\end{array}\right) B_{j}(\omega) \| \\
& +\left.\max _{\omega \in \mathbb{T}} 2^{-4}|| \cos ^{2}\left(\frac{\omega}{4}\right)\right|^{2} \\
& +\left|1+\left(\max _{\omega \in[-\pi, \pi]} B_{j}(\omega)\right) \sum_{j=1}^{l}\left(\begin{array}{c}
m+l \\
j
\end{array}\right)\right|^{2}\left|<2^{-4}\right| 1 \\
& \left.+\mid \frac{1}{2}\right)\left.^{m+l-1}(2)^{m+l}\right|^{2} \mid=\frac{5}{8} .
\end{aligned}
$$

Bringing Lemmas 1 and 2 together yields

$$
\begin{aligned}
\rho\left(\tau^{m, l, \alpha}(\omega)\right) & \leqslant \rho_{4}\left(\tau^{m, l, \alpha}(\omega), \infty\right) \\
& =\rho_{0}\left(2^{-2} T_{\tau^{m, l, \alpha}(\omega)}, \infty\right)<1 .
\end{aligned}
$$

Thus, by Lemma 3, the cascade algorithm associated with the mark $\tau^{m, l, \alpha}(\omega)$ converges in the space $L_{2, \infty}(\mathbb{R})$.

\section{Symmetry}

Symmetric coefficients of the mark are of great significance in image processing. The following lemma is helpful for the demonstration of symmetry. 
Lemma 10. For $m, l \in \mathbb{Z}^{+}, \alpha \geq 0, j=0,1, \ldots, l, z=e^{-i(\omega / 2)}$, we derive

$$
=\sum_{k=0}^{m+l} b_{k}^{j, m, l, \alpha}\left(z^{-2}+z^{2}\right)^{k}
$$

$$
\begin{gathered}
\prod_{k=0}^{j-1}\left(\frac{1}{2}+\frac{1}{4}\left(z^{-2}+z^{2}\right)+k \alpha\right) \\
\cdot \prod_{k=0}^{m+l-j-1}\left(\frac{1}{2}-\frac{1}{4}\left(z^{-2}+z^{2}\right)+k \alpha\right) \\
b_{k}^{j, m, l, \alpha}=\frac{A_{k}}{A}, \quad k=0,1, \ldots, m+l,
\end{gathered}
$$

$$
\begin{aligned}
& A=\left|\begin{array}{ccccc}
1 & \frac{1}{2} & \left(\frac{1}{2}\right)^{2} & \cdots & \left(\frac{1}{2}\right)^{m+l} \\
1 & \left(\frac{1}{2}\right)^{2} & \left(\left(\frac{1}{2}\right)^{2}\right)^{2} & \cdots & \left(\left(\frac{1}{2}\right)^{2}\right)^{m+l} \\
\cdots & \cdots & \cdots & \cdots & \cdots \\
1 & \left(\frac{1}{2}\right)^{m+l+1} & \left(\left(\frac{1}{2}\right)^{m+l+1}\right)^{2} & \cdots & \left(\left(\frac{1}{2}\right)^{m+l+1}\right)^{m+l}
\end{array}\right|,
\end{aligned}
$$

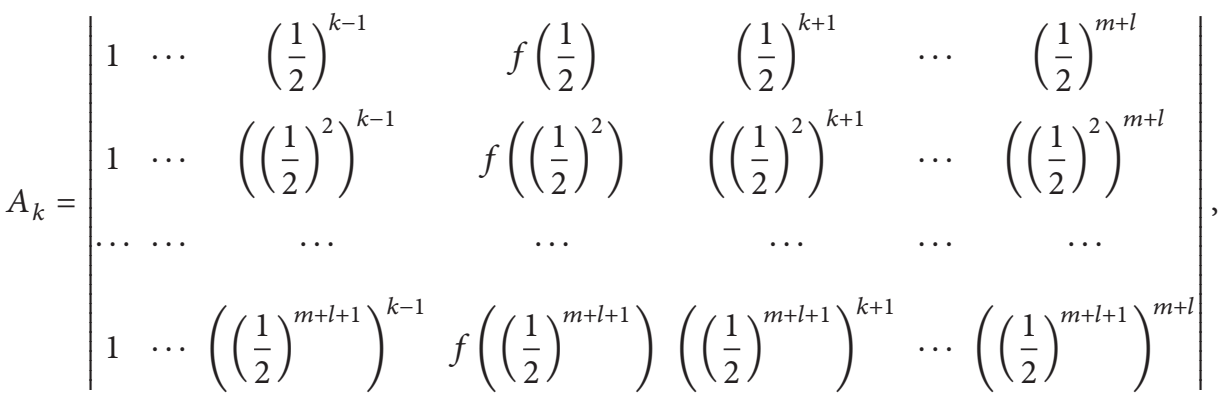

where

$$
\begin{array}{r}
f(x)=\prod_{k=0}^{j-1}\left(\frac{1}{2}+\frac{1}{4} x+k \alpha\right) \prod_{k=0}^{m+l-j-1}\left(\frac{1}{2}-\frac{1}{4} x+k \alpha\right), \\
x \in[-2,2] .
\end{array}
$$

Proof. Set $x(z)=z^{-2}+z$; then $x \in[-2,2]$ and

$$
\begin{aligned}
& \prod_{k=0}^{j-1}\left(\frac{1}{2}+\frac{1}{4} x+k \alpha\right) \prod_{k=0}^{m+l-j-1}\left(\frac{1}{2}-\frac{1}{4} x+k \alpha\right) \\
& \quad=\sum_{k=0}^{m+l} b_{k}^{j, m, l, \alpha} x^{k} .
\end{aligned}
$$

Fixing $x=1 / 2,(1 / 2)^{2}, \ldots,(1 / 2)^{m+l+1}$, by using (54), we obtain

$$
\begin{aligned}
& \left(\frac{1}{2}\right)^{0} b_{0}^{j, m, l, \alpha}+\left(\frac{1}{2}\right)^{1} b_{1}^{j, m, l, \alpha}+\cdots \\
& +\left(\frac{1}{2}\right)^{m+l} b_{m+l}^{j, m, l, \alpha}=f\left(\frac{1}{2}\right) \\
& \left(\left(\frac{1}{2}\right)^{2}\right)^{0} b_{0}^{j, m, l, \alpha}+\left(\left(\frac{1}{2}\right)^{2}\right)^{1} b_{1}^{j, m, l, \alpha}+\cdots \\
& +\left(\left(\frac{1}{2}\right)^{2}\right)^{m+l} b_{m+l}^{j, m, l, \alpha}=f\left(\left(\frac{1}{2}\right)^{2}\right) \\
& \left(\left(\frac{1}{2}\right)^{m+l+1}\right)^{0} b_{0}^{j, m, l, \alpha}+\left(\left(\frac{1}{2}\right)^{m+l+1}\right)^{1} b_{1}^{j, m, l, l \alpha}+\cdots \\
& +\left(\left(\frac{1}{2}\right)^{m+l+1}\right)^{m+l} b_{m+l}^{j, m, l, \alpha}=f\left(\left(\frac{1}{2}\right)^{m+l+1}\right) .
\end{aligned}
$$


Since coefficient determinant of (55)

A

$$
=\left|\begin{array}{ccccc}
1 & \frac{1}{2} & \left(\frac{1}{2}\right)^{2} & \cdots & \left(\frac{1}{2}\right)^{m+l} \\
1 & \left(\frac{1}{2}\right)^{2} & \left(\left(\frac{1}{2}\right)^{2}\right)^{2} & \cdots & \left(\left(\frac{1}{2}\right)^{2}\right)^{m+l} \\
\cdots & \cdots & \cdots & \cdots & \cdots \\
1 & \left(\frac{1}{2}\right)^{m+l+1} & \left(\left(\frac{1}{2}\right)^{m+l+1}\right)^{2} & \cdots & \left(\left(\frac{1}{2}\right)^{m+l+1}\right)^{m+l}
\end{array}\right|
$$$$
\neq 0,
$$

applying Cramer's Rule yields

$$
b_{k}^{j, m, l, \alpha}=\frac{A_{k}}{A}, \quad k=0,1, \ldots, m+l,
$$

where

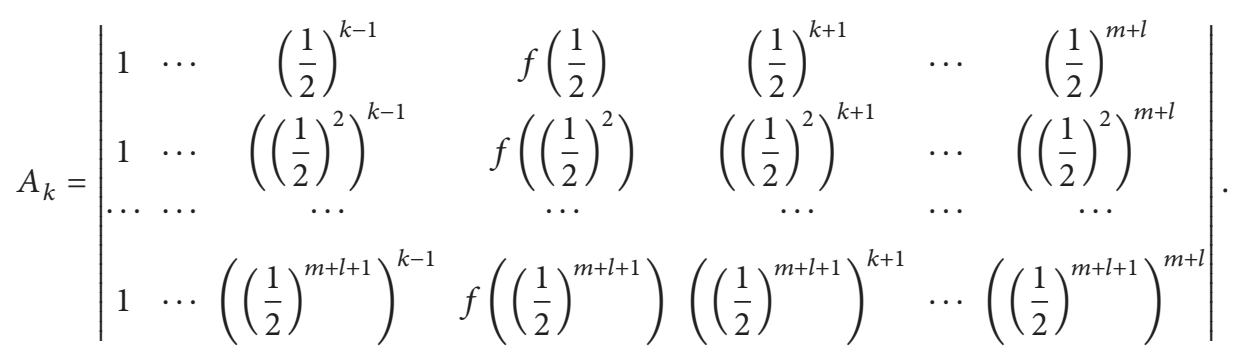

In the following, we will give a symmetry proof.

Proof. Let

Theorem 11. For two positive integers $l, m$, satisfying $l<m-5$, let $\tau^{m, l, \alpha}(\omega)$ be mask (2); then the coefficients of the mask are symmetric.

$$
M_{0}^{m, l, \alpha}(\omega)=\frac{\sum_{j=0}^{l}\left(\begin{array}{c}
m+l \\
j
\end{array}\right)\left(\prod_{k=0}^{j-1}\left(\cos ^{2}(\omega / 2)+k \alpha\right) \prod_{k=0}^{m+l-j-1}\left(\sin ^{2}(\omega / 2)+k \alpha\right)\right)}{\prod_{k=1}^{m+l-1}(1+k \alpha)}
$$

and then

$$
\tau^{m, l, \alpha}(\omega)=\cos ^{2}\left(\frac{\omega}{2}\right)\left(\cos ^{2}\left(\frac{\omega}{4}\right)-M_{0}^{m, l, \alpha}(\omega)\right) .
$$

Since $\sin ^{2}(\omega / 2)=1 / 2-(1 / 4)\left(e^{i \omega}+e^{-i \omega}\right), \cos ^{2}(\omega / 2)=1 / 2+$ $(1 / 4)\left(e^{i \omega}+e^{-i \omega}\right)$, we set $z=e^{-i(\omega / 2)}$ and obtain

$$
\begin{aligned}
M_{0}^{m, l, \alpha}(\omega) & =\frac{\sum_{j=0}^{l}\left(\begin{array}{c}
m+l \\
j
\end{array}\right)\left(\prod_{k=0}^{j-1}\left(\cos ^{2}(\omega / 2)+k \alpha\right) \prod_{k=0}^{m+l-j-1}\left(\sin ^{2}(\omega / 2)+k \alpha\right)\right)}{\prod_{k=1}^{m+l-1}(1+k \alpha)} \\
& =\frac{\sum_{j=0}^{l}\left(\begin{array}{c}
m+l \\
j
\end{array}\right)\left(\prod_{k=0}^{j-1}\left(1 / 2+(1 / 4)\left(e^{k \omega}+e^{-k \omega}\right)+e^{-k \omega}+k \alpha\right) \prod_{k=0}^{m+l-j-1}\left(1 / 2-(1 / 4)\left(e^{k \omega}+e^{-k \omega}\right)+k \alpha\right)\right)}{\prod_{k=1}^{m+l-1}(1+k \alpha)} \\
& =\frac{\sum_{j=0}^{l}\left(\begin{array}{c}
m+l \\
j
\end{array}\right)\left(\prod_{k=0}^{j-1}\left(1 / 2+(1 / 4)\left(z^{-2}+z^{2}\right)+k \alpha\right) \prod_{k=0}^{m+l-j-1}\left(1 / 2-(1 / 4)\left(z^{-2}+z^{2}\right)+k \alpha\right)\right)}{\prod_{k=1}^{m+l-1}(1+k \alpha)} .
\end{aligned}
$$


Let $a_{j}^{m, l, \alpha}=\left(\begin{array}{c}m+l \\ j\end{array}\right) / \prod_{i=1}^{m+l-1}(1+i \alpha)$; by using Lemma 10, one can obtain that

$$
\begin{aligned}
M_{0}^{m, l, \alpha}(\omega) & =\frac{\sum_{j=0}^{l}\left(\begin{array}{c}
m+l \\
j
\end{array}\right)\left(\prod_{k=0}^{j-1}\left(1 / 2+(1 / 4)\left(z^{-2}+z^{2}\right)+k \alpha\right) \prod_{k=0}^{m+l-j-1}\left(1 / 2-(1 / 4)\left(z^{-2}+z^{2}\right)+k \alpha\right)\right)}{\prod_{k=1}^{m+l-1}(1+k \alpha)} \\
& =\sum_{j=0}^{l} a_{j}^{m, l, \alpha} \sum_{k=0}^{m+l} b_{k}^{j, m, l, \alpha}\left(z+z^{-1}\right)^{k}=\sum_{j=0}^{l} \sum_{k=0}^{m+l} a_{j}^{m, l, \alpha} b_{k}^{j, m, l, \alpha}\left(z+z^{-1}\right)^{k},
\end{aligned}
$$

where $\left\{b_{k}^{j, m, l, \alpha}\right\}_{k=0}^{m+l}$ is (51) in Lemma 10. Let $c_{k}^{j, m, l, \alpha}=$ $a_{j}^{m, l, \alpha} b_{k}^{j, m, l, \alpha}$ and $d_{k}^{m, l, \alpha}=\sum_{j=0}^{l} c_{k}^{j, m, l, \alpha}$; then

$$
\begin{aligned}
M_{0}^{m, l, \alpha}(\omega) & =\sum_{j=0}^{l} \sum_{k=0}^{m+l} a_{j}^{m, l, \alpha} b_{k}^{j, m, l, \alpha}\left(z+z^{-1}\right)^{k} \\
& =\sum_{j=0}^{l} \sum_{k=0}^{m+l} c_{k}^{j, m, l, \alpha}\left(z+z^{-1}\right)^{k}
\end{aligned}
$$

$$
\begin{aligned}
& =\sum_{k=0}^{m+l}\left(\sum_{j=0}^{l} c_{k}^{j, m, l, \alpha}\right)\left(z+z^{-1}\right)^{k} \\
& =\sum_{k=0}^{m+l} d_{k}^{m, l, \alpha}\left(z+z^{-1}\right)^{k} .
\end{aligned}
$$

We consider two cases. Suppose that $m+l$ is an even number. Applying

$$
\left(z+z^{-1}\right)^{k}= \begin{cases}\sum_{j=0}^{(k-1) / 2}\left(\begin{array}{l}
k \\
j
\end{array}\right)\left(z^{k-2 j}+z^{-(k-2 j)}\right), & \text { if } k \text { is an odd number } \\
\sum_{j=0}^{k / 2-1}\left(\begin{array}{l}
k \\
j
\end{array}\right)\left(z^{k-2 j}+z^{-(k-2 j)}\right)+\left(\begin{array}{l}
k \\
\frac{k}{2}
\end{array}\right) z^{0}, & \text { else }\end{cases}
$$

yields

$$
\begin{aligned}
& M_{0}^{m, l, \alpha}(\omega)=\sum_{k=0}^{m+l} d_{k}^{m, l, \alpha}\left(z+z^{-1}\right)^{k} \\
& =\sum_{j=0}^{(m+l-2) / 2} d_{2 j+1}^{m, l, \alpha} \sum_{k=0}^{j}\left(\begin{array}{c}
2 j+1 \\
k
\end{array}\right)\left(z^{2(j-k)+1}\right. \\
& \left.+z^{-(2(j-k)+1)}\right)+\sum_{j=0}^{(m+l) / 2} d_{2 j}^{m, l, \alpha} \sum_{k=0}^{j-1}\left(\begin{array}{c}
2 j \\
k
\end{array}\right)\left(z^{2(j-k)}\right. \\
& \left.+z^{-(2(j-k))}\right)+\sum_{j=0}^{(m+l) / 2} d_{2 j}^{m, l, \alpha}\left(\begin{array}{c}
2 j \\
j
\end{array}\right) \\
& =\sum_{j=0}^{(m+l-2) / 2} \sum_{k=0}^{j}\left(\begin{array}{c}
2 j+1 \\
k
\end{array}\right) d_{2 j+1}^{m, l, \alpha}\left(z^{2(j-k)+1}\right. \\
& \left.+z^{-(2(j-k)+1)}\right)+\sum_{j=0}^{(m+l) / 2} \sum_{k=0}^{j-1}\left(\begin{array}{c}
2 j \\
k
\end{array}\right) d_{2 j}^{m, l, \alpha}\left(z^{2(j-k)}\right. \\
& \left.+z^{-(2(j-k))}\right)+\sum_{j=0}^{(m+l) / 2} d_{2 j}^{m, l, \alpha}\left(\begin{array}{c}
2 j \\
j
\end{array}\right)=\sum_{k=1}^{m+l} q_{k}^{m, l, \alpha}\left(z^{k}\right. \\
& \left.+z^{-k}\right)+h_{0} z^{0}, \sum_{k=1}^{(m-1)}
\end{aligned}
$$

where $q_{0}=\sum_{j=0}^{(m+l) / 2}\left(\begin{array}{c}2 j \\ j\end{array}\right) d_{2 j}^{m, l, \alpha}$,

$$
\begin{aligned}
& q_{k} \\
& =\left\{\begin{array}{cl}
\sum_{\substack{j-i=(k-1) / 2 \\
j \in\{0,1, \ldots,(m+l-2) / 2\}}}\left(\begin{array}{c}
2 j+1 \\
i
\end{array}\right) d_{2 j+1}^{m, l, \alpha}, & \text { if } k \text { is an odd number } \\
\sum_{\substack{j-i=k / 2 \\
j \in\{0,1, \ldots,(m+l) / 2\}}}\left(\begin{array}{c}
2 j \\
i
\end{array}\right) d_{2 j}^{m, l, \alpha}, & \text { else. }
\end{array}\right.
\end{aligned}
$$

Thus,

$$
\begin{aligned}
\tau^{m, l, \alpha} & (\omega)=\cos ^{2}\left(\frac{\omega}{2}\right)\left(\cos ^{2}\left(\frac{\omega}{4}\right)-M_{0}^{m, l, \alpha}(\omega)\right) \\
= & \left(\frac{1}{2}+\frac{1}{4}\left(z^{-2}+z^{2}\right)\right)\left(\frac{1}{2}+\frac{1}{4}\left(z^{-1}+z\right)\right. \\
- & \left.\left(\sum_{k=1}^{m+l} q_{k}^{m, l, \alpha}\left(z^{k}+z^{-k}\right)+q_{0} z^{0}\right)\right) \\
= & \sum_{k=1}^{m+l+2} h_{k}^{m, l, \alpha}\left(z^{k}+z^{-k}\right)+h_{0} z^{0},
\end{aligned}
$$


where $h_{0}=(1 / 2)\left(q_{0}+1 / 2\right)$,

$$
\begin{aligned}
& h_{k} \\
& = \begin{cases}\frac{1}{2}\left(q_{i}+\frac{1}{4}\right), & k= \pm 1 \\
\frac{1}{4} q_{i-2}+\frac{1}{2} q_{i}, & k= \pm 2, \pm 3, \ldots, \pm(m+l) \\
\frac{1}{4} q_{i-2}, & k= \pm(m+l+1), \pm(m+l+2) .\end{cases}
\end{aligned}
$$

Suppose, on the other hand, that $m+l$ is an odd number. It holds

$$
\begin{aligned}
& M_{0}^{m, l, \alpha}(\omega)=\sum_{k=0}^{m+l} d_{k}^{m, l, \alpha}\left(z+z^{-1}\right)^{k} \\
& =\sum_{j=0}^{(m+l-1) / 2} d_{2 j+1}^{m, l, \alpha} \sum_{k=0}^{j}\left(\begin{array}{c}
2 j+1 \\
k
\end{array}\right)\left(z^{2(j-k)+1}\right. \\
& \left.+z^{-(2(j-k)+1)}\right)+\sum_{j=0}^{(m+l-1) / 2} d_{2 j}^{m, l, \alpha} \sum_{k=0}^{j-1}\left(\begin{array}{c}
2 j \\
k
\end{array}\right)\left(z^{2(j-k)}\right. \\
& \left.+z^{-(2(j-k))}\right)+\sum_{j=0}^{(m+l-1) / 2} d_{2 j}^{m, l, \alpha}\left(\begin{array}{c}
2 j \\
j
\end{array}\right) \\
& =\sum_{j=0}^{(m+l-1) / 2} \sum_{k=0}^{j}\left(\begin{array}{c}
2 j+1 \\
k
\end{array}\right) d_{2 j+1}^{m, l, \alpha}\left(z^{2(j-k)+1}\right. \\
& \left.+z^{-(2(j-k)+1)}\right)+\sum_{j=0}^{(m+l-1) / 2} \sum_{k=0}^{j-1}\left(\begin{array}{c}
2 j \\
k
\end{array}\right) d_{2 j}^{m, l, \alpha}\left(z^{2(j-k)}\right. \\
& \left.+z^{-(2(j-k))}\right)+\sum_{j=0}^{(m+l-1) / 2} d_{2 j}^{m, l, \alpha}\left(\begin{array}{c}
2 j \\
j
\end{array}\right)=\sum_{k=1}^{m+l} q_{k}^{m, l, \alpha}\left(z^{k}\right.
\end{aligned}
$$

where $q_{0}=\sum_{j=0}^{(m+l-1) / 2}\left(\begin{array}{c}2 j \\ j\end{array}\right) d_{2 j}^{m, l, \alpha}$,

$$
\begin{aligned}
& q_{k} \\
& =\left\{\begin{array}{cl}
\sum_{\substack{j-i=(k-1) / 2 \\
j \in\{0,1, \ldots,(m+l-1) / 2\}}}\left(\begin{array}{c}
2 j+1 \\
i
\end{array}\right) d_{2 j+1}^{m, l, \alpha}, & \text { if } k \text { is an odd number } \\
\sum_{\substack{j-i=k / 2 \\
j \in\{0,1, \ldots,(m+l-1) / 2\}}}\left(\begin{array}{c}
2 j \\
i
\end{array}\right) d_{2 j}^{m, l, \alpha}, & \text { else. }
\end{array}\right.
\end{aligned}
$$

Therefore,

$$
\begin{gathered}
\tau^{m, l, \alpha}(\omega)=\cos ^{2}\left(\frac{\omega}{2}\right)\left(\cos ^{2}\left(\frac{\omega}{4}\right)-M_{0}^{m, l, \alpha}(\omega)\right) \\
=\left(\frac{1}{2}+\frac{1}{4}\left(z^{-2}+z^{2}\right)\right)\left(\frac{1}{2}+\frac{1}{4}\left(z^{-1}+z\right)\right. \\
\left.-\left(\sum_{k=1}^{m+l} q_{k}^{m, l, \alpha}\left(z^{k}+z^{-k}\right)+q_{0} z^{0}\right)\right) \\
=\sum_{k=1}^{m+l+2} h_{k}^{m, l, \alpha}\left(z^{k}+z^{-k}\right)+h_{0} z^{0},
\end{gathered}
$$

where $h_{0}=(1 / 2)\left(q_{0}+1 / 2\right)$,

$$
\begin{aligned}
& h_{k} \\
& = \begin{cases}\frac{1}{2}\left(q_{i}+\frac{1}{4}\right), & k= \pm 1 \\
\frac{1}{4} q_{i-2}+\frac{1}{2} q_{i}, & k= \pm 2, \pm 3, \ldots, \pm(m+l) \\
\frac{1}{4} q_{i-2}, & k= \pm(m+l+1), \pm(m+l+2) .\end{cases}
\end{aligned}
$$

This establishes the proof.

\section{Regularity and Approximation Orders}

This section is devoted to analysis of the regularity and approximation orders of refinable functions $\phi^{m, l, \alpha}$ with the mask $\tau^{m, l, \alpha}(\omega)$ defined by (2) in the following theorem.

Theorem 12. For two positive integers $l, m$, satisfying $l<m-5$, let $\phi^{m, l, \alpha}$ be refinable functions with the mask $\tau^{m, l, \alpha}(\omega)$. Then

$$
\left|\widehat{\phi}_{0}^{m, l, \alpha}\right| \leq C(1+|\omega|)^{-2+\mathscr{K}}
$$

where $\mathscr{K}=\log \left(\left|T^{m, l, \alpha}(2 \pi / 3)\right|\right) / \log 2$, and the decay rate $\beta_{0}^{m, l, \alpha}=2-\log \left(\left|T^{m, l, \alpha}(2 \pi / 3)\right|\right) / \log 2$ is optimal. As a result, $\phi^{m, l, \alpha} \in C^{\gamma_{0}^{m, l, \alpha}}$, where $\gamma_{0}^{m, l, \alpha} \geq \beta_{0}^{m, l, \alpha}-1-\varepsilon$, for any small enough $\varepsilon>0$.

Proof. It is obvious that $\left|\tau^{m, l, \alpha}(\omega)\right|=\cos ^{2}(\omega / 2)\left|T^{m, l, \alpha}(\omega)\right|, \omega \epsilon$ $[-\pi, \pi]$. We claim that

$$
\left|T^{m, l, \alpha}(\omega)\right| \leq\left|T^{m, l, \alpha}\left(\frac{2 \pi}{3}\right)\right|, \quad \text { for }|\omega| \in\left[0, \frac{2 \pi}{3}\right] .
$$

Indeed, $T^{m, l, \alpha}(\omega)$ is a continuous function on $[-2 \pi / 3,2 \pi / 3]$ and is differentiable on $(-2 \pi / 3,2 \pi / 3)$. The maximum value of $T^{m, l, \alpha}(\omega)$ on $[-2 \pi / 3,2 \pi / 3]$ can be derived as follows: we find that $\omega=0$ is the only zero of equation $\left[T^{m, l, \alpha}(\omega)\right]^{\prime}=0$. Obviously, $T^{m, l, \alpha}(0)$ is the minimum of $T^{m, l, \alpha}(\omega)$ on $[-2 \pi / 3,2 \pi / 3]$. 
Consequently, $T^{m, l, \alpha}(0) \leq T^{m, l, \alpha}( \pm 2 \pi / 3)$. Here, $T^{m, l, \alpha}(\omega)$ is an even function. Hence, (74) holds. Next we show

$$
\begin{aligned}
\left|T^{m, l, \alpha}(\omega) T^{m, l, \alpha}(2 \omega)\right| \leq\left|T^{m, l, \alpha}\left(\frac{2 \pi}{3}\right)\right|^{2}, & \\
& \text { for }|\omega| \in\left[\frac{2 \pi}{3}, \pi\right] .
\end{aligned}
$$

Here, $T^{m, l, \alpha}(\omega) T^{m, l, \alpha}(2 \omega)$ is a continuous function on $|\omega| \epsilon$ $[2 \pi / 3, \pi]$ and is differentiable on $|\omega| \in(2 \pi / 3, \pi)$. Since equation $\left[T^{m, l, \alpha}(\omega) T^{m, l, \alpha}(2 \omega)\right]^{\prime}=0$ has no zeros on $|\omega| \epsilon$ $[2 \pi / 3, \pi]$, we are required to compare $T^{m, l, \alpha}(\pi) T^{m, l, \alpha}(2 \pi)$ with $T^{m, l, \alpha}(2 \pi / 3) T^{m, l, \alpha}(4 \pi / 3)$. Because of

$$
\begin{aligned}
& T^{m, l, \alpha}(\pi) T^{m, l, \alpha}(2 \pi)=0, \\
& T^{m, l, \alpha}\left(\frac{2 \pi}{3}\right) T^{m, l, \alpha}\left(\frac{4 \pi}{3}\right) \\
& \quad=T^{m, l, \alpha}\left(\frac{2 \pi}{3}\right) T^{m, l, \alpha}\left(-\frac{2 \pi}{3}\right)=\left(T^{m, l, \alpha}\left(\frac{2 \pi}{3}\right)\right)^{2},
\end{aligned}
$$

we get $\left(T^{m, l, \alpha}(2 \pi / 3)\right)^{2} \geq 0$. Thus, (75) holds. By Lemma 4 , $\widehat{\phi}_{0}^{m, l, \alpha}$ satisfies

$$
\left|\widehat{\phi}^{m, l, \alpha}\right| \leq C(1+|\omega|)^{-2+\mathscr{K}}
$$

where $\mathscr{K}=\log \left(\left|T^{m, l, \alpha}(2 \pi / 3)\right|\right) / \log 2$ and the decay rate $\beta_{0}^{m, l, \alpha}=2-\log \left(\left|T^{m, l, \alpha}(2 \pi / 3)\right|\right) / \log 2$ is optimal. As a result, $\phi^{m, l, \alpha} \in C^{\gamma_{0}^{m, l \alpha}}$, where $\gamma_{0}^{m, l, \alpha} \geq \beta_{0}^{m, l, \alpha}-1-\varepsilon$, for any small enough $\varepsilon>0$.

Theorem 13. For two positive integers $l$, $m$, satisfying $l<m-5$, let $\phi^{m, l, \alpha}$ be refinable functions with the mask $\tau^{m, l, \alpha}(\omega)$. Then $\phi^{m, l, \alpha}$ provides the approximation orders $2 l+2$.
Proof. In fact, the approximation orders of $1-\left|\tau^{m, l, \alpha}(\omega)\right|^{2}$ and $\tau^{m, l, \alpha}(\omega)$ are independent on $\alpha$. For convenience, we set $\alpha=0$. Following [8], let

$$
R_{m, l}(y)=\sum_{j=0}^{l}\left(\begin{array}{c}
m+l \\
j
\end{array}\right) y^{j}(1-y)^{m+l-j},
$$

where $y=\cos ^{2}(\omega / 2)$. Since

$$
\begin{aligned}
(1 & \left.-\left|R_{m, l} \cos ^{2}\left(\frac{\omega}{2}\right)\right|^{2}\right)^{\prime} \\
& =1-\cos ^{4}\left(\frac{\omega}{2}\right)\left(\cos ^{2}\left(\frac{\omega}{4}\right)-R_{m, l}(y)\right)^{2},
\end{aligned}
$$

$\sin ^{2}(\omega / 2)$ is equal to 1 when $\omega=\pi$, and $\cos ^{2}(\omega / 2)$ has zero of order 4 , we conclude that

$$
\begin{aligned}
1-\left|R_{m, l} \cos ^{2}\left(\frac{\omega}{2}\right)\right|^{2} & =O\left(|\omega|^{2 l+3}\right), \\
\tau^{m, l, \alpha}(\omega) & =O\left(|\omega|^{2 l+2}\right) .
\end{aligned}
$$

For

$$
\min \{2 l+3,2 l+2\}=2 l+2,
$$

then $\phi^{m, l, \alpha}$ provides the approximation orders $2 l+2$.

\section{Tight and Sibling Frames}

In this section, tight and sibling frames are constructed in the following theorem. At the same time, the interorthogonality of sibling frames is implemented.

Theorem 14. For two positive integers $l, m$, satisfying $l<$ $m-5$, let $\tau^{m, l, \alpha}(\omega)$ be mask (2), then there exist compactly supported sibling frames $\left\{\psi_{1}, \psi_{2}\right\},\left\{\widetilde{\psi}_{1}, \widetilde{\psi}_{2}\right\}$ defined in (19), with the property that all of the four functions have 2 vanishing moments. Furthermore, all of the four functions are symmetric.

Proof. Let $z=e^{-i(\omega / 2)}$ :

$$
\begin{aligned}
P_{0}(z) & =z^{-1}\left(\left(\frac{1}{2}+\frac{1}{4}\left(z^{-2}+z^{2}\right)\right)(1\right. \\
& \left.\left.-\frac{\left(4-4((1+z) / 2)^{2} z^{-1}\right) \sum_{j=0}^{l}\left(\begin{array}{c}
m+l \\
j
\end{array}\right)\left(\prod_{k=0}^{j-1}\left(1 / 2+(1 / 4)\left(z^{-2}+z^{2}\right)+k \alpha\right) \prod_{k=1}^{m+l-j-1}\left(1 / 2-(1 / 4)\left(z^{-2}+z^{2}\right)+k \alpha\right)\right)}{\prod_{k=1}^{m+l-1}(1+k \alpha)}\right)\right) .
\end{aligned}
$$

We get

$$
\begin{gathered}
\tau^{m, l, \alpha}(\omega)=\cos ^{2}\left(\frac{\omega}{2}\right)\left(\cos ^{2}\left(\frac{\omega}{4}\right)-\frac{\sum_{j=0}^{l}\left(\begin{array}{c}
m+l \\
j
\end{array}\right)\left(\prod_{k=0}^{j-1}\left(\cos ^{2}(\omega / 2)+k \alpha\right) \prod_{k=0}^{m+l-j-1}\left(\sin ^{2}(\omega / 2)+k \alpha\right)\right)}{\prod_{k=1}^{m+l-1}(1+k \alpha)}\right)=\cos ^{2}\left(\frac{\omega}{2}\right) \\
\cdot\left(\cos ^{2}\left(\frac{\omega}{4}\right)-\frac{\sin ^{2}(\omega / 2) \sum_{j=0}^{l}\left(\begin{array}{c}
m+l \\
j
\end{array}\right)\left(\prod_{k=0}^{j-1}\left(\cos ^{2}(\omega / 2)+k \alpha\right) \prod_{k=1}^{m+l-j-1}\left(\sin ^{2}(\omega / 2)+k \alpha\right)\right)}{\prod_{k=1}^{m+l-1}(1+k \alpha)}\right)=\cos ^{2}\left(\frac{\omega}{2}\right)
\end{gathered}
$$




$$
\begin{aligned}
& \cdot\left(\cos ^{2}\left(\frac{\omega}{4}\right)-\frac{\left(4 \cos ^{2}(\omega / 4)-4 \cos ^{4}(\omega / 4)\right) \sum_{j=0}^{l}\left(\begin{array}{c}
m+l \\
j
\end{array}\right)\left(\prod_{k=0}^{j-1}\left(\cos ^{2}(\omega / 2)+k \alpha\right) \prod_{k=1}^{m+l-j-1}\left(\sin ^{2}(\omega / 2)+k \alpha\right)\right)}{\prod_{k=1}^{m+l-1}(1+k \alpha)}\right) \\
& =\cos ^{2}\left(\frac{\omega}{4}\right) \\
& \cdot\left(\cos ^{2}\left(\frac{\omega}{2}\right)\left(1-\frac{\left(4-4 \cos ^{2}(\omega / 4)\right) \sum_{j=0}^{l}\left(\begin{array}{c}
m+l \\
j
\end{array}\right)\left(\prod_{k=0}^{j-1}\left(\cos ^{2}(\omega / 2)+k \alpha\right) \prod_{k=1}^{m+l-j-1}\left(\sin ^{2}(\omega / 2)+k \alpha\right)\right)}{\prod_{k=1}^{m+l-1}(1+k \alpha)}\right)\right) \\
& =\left(\frac{1+z}{2}\right)^{2} P_{0}(z) .
\end{aligned}
$$

Here, $P_{0}(-1) \neq 0$.

Obviously,

$$
\widehat{\phi}(0)=1 .
$$

In [2], another way of looking at this is that the decay condition

$$
|\psi| \leq C_{1}(1+|x|)^{-1-\epsilon}, \quad x \in \mathbb{R},
$$

for some $\epsilon>0$ and $0<C_{1}<\infty$, automatically implies that $\widehat{\psi} \in \operatorname{Lip} \alpha$, for $\alpha<\epsilon$.

Applying Theorem 12 yields

$$
\phi \in \operatorname{Lip} \alpha, \quad \text { for } \alpha<\epsilon
$$

Then, it is obvious that

$$
\phi \text { is piecewise } \operatorname{Lip} \alpha, \quad \text { for } \alpha>0 \text {. }
$$

Hence, by Lemma 5 and (83)-(87), there exist compactly supported sibling frames $\left\{\psi_{1}, \psi_{2}\right\},\left\{\widetilde{\psi}_{1}, \widetilde{\psi}_{2}\right\}$ defined in (19).

Theorem 15. For two positive integers $l, m$, satisfying $l<$ $m-5$, let $\tau^{m, l, \alpha}(\omega)$ be mask (2); then there exists a pair of sibling frames $\left(\psi_{1}, \psi_{2}\right)$ and $\left(\widetilde{\psi}_{1}, \widetilde{\psi}_{2}\right)$, with the property that all of the four functions have compact support and the maximum number 2 of vanishing moments. And, $\left(\psi_{1}, \psi_{2}\right)$ is interorthogonal.

Proof. We claim that condition (8)-(16) holds. Indeed, the refinable functions with the masks $\tau^{m, l, \alpha}(\omega)$, which satisfy (8)-(16) as shown in Theorem 14, have been proved. Applying Lemma 6 yields the conclusion.

Theorem 16. For two positive integers $l, m$, satisfying $l<m-5$, let $\tau^{m, l, \alpha}(\omega)$ be mask (2): $Q_{t}=(z / \sqrt{S(-1)}) S(z) P(-1 / z)$, where $S$ is Laurent polynomial. Then $\widetilde{\psi}_{t}(\omega)=Q_{t}(z) \widetilde{\phi}(\omega / 2)$ defines a tight frame of $L^{2}$ which is associated with the same VMR function $S$.

Proof. Since $\tau^{m, l, \alpha}(\omega)$ is mask (2), by Theorem 14, there exist compactly supported sibling frames $\{\psi\},\{\widetilde{\psi}\}$. Then let $\{\psi\}$, $\{\widetilde{\psi}\}$ associate with a VMR function $S$, where $S$ is Laurent polynomial.

Applying Lemma 7, then $\left\{\psi_{t}\right\}$ construct a tight frame of $L^{2}$ which is associated with the same VMR function $S$.

\section{Numerical Example}

Consider Theorem 16 with the condition $S(z)$ as follows.

Example 1 (see Figure 1). Take

$$
\tau^{7,1,0.05}(\omega)=\cos ^{2}\left(\frac{\omega}{2}\right)\left(\cos ^{2}\left(\frac{\omega}{4}\right)-\frac{\sum_{j=0}^{1}\left(\begin{array}{l}
8 \\
j
\end{array}\right)\left(\prod_{k=0}^{j-1}\left(\cos ^{2}(\omega / 2)+0.05 k\right) \prod_{k=0}^{7-j}\left(\sin ^{2}(\omega / 2)+0.05 k\right)\right)}{\prod_{k=1}^{7}(1+0.05 k)}\right) .
$$

The corresponding refinable function is order 4 . Let $S(z)=1$, and get $Q_{t}$ in Theorem 16.

\section{Example 2 (see Figure 2). Take}

$$
\tau^{12,1,0.05}(\omega)=\cos ^{2}\left(\frac{\omega}{2}\right)\left(\cos ^{2}\left(\frac{\omega}{4}\right)-\frac{\sum_{j=0}^{1}\left(\begin{array}{c}
13 \\
j
\end{array}\right)\left(\prod_{k=0}^{j-1}\left(\cos ^{2}(\omega / 2)+0.05 k\right) \prod_{k=0}^{12-j}\left(\sin ^{2}(\omega / 2)+0.05 k\right)\right)}{\prod_{k=1}^{12}(1+0.05 k)}\right) .
$$

The corresponding refinable function is order 4 . Let $S(z)=1$, and get $Q_{t}$ in Theorem 16 .
In Figures 1 and 2, we give the examples of tight and sibling frames with one generator; at the same time, their 


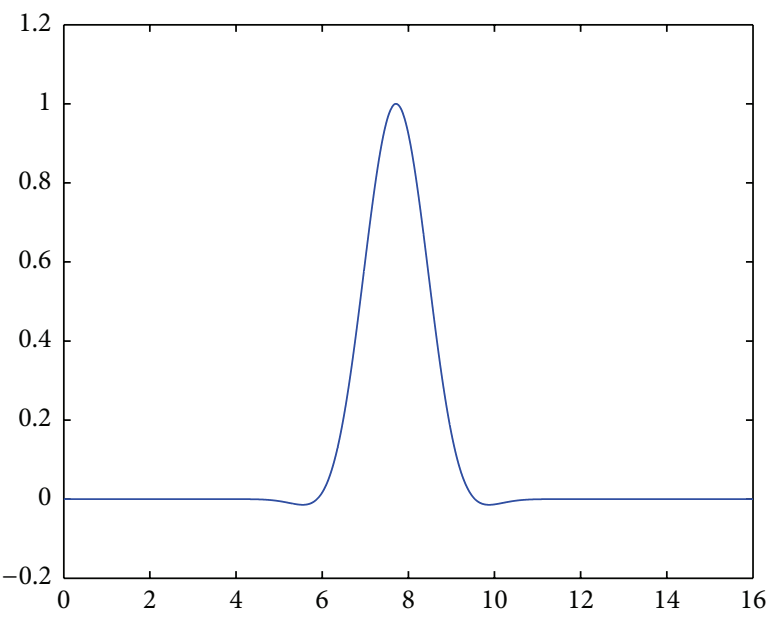

(a)

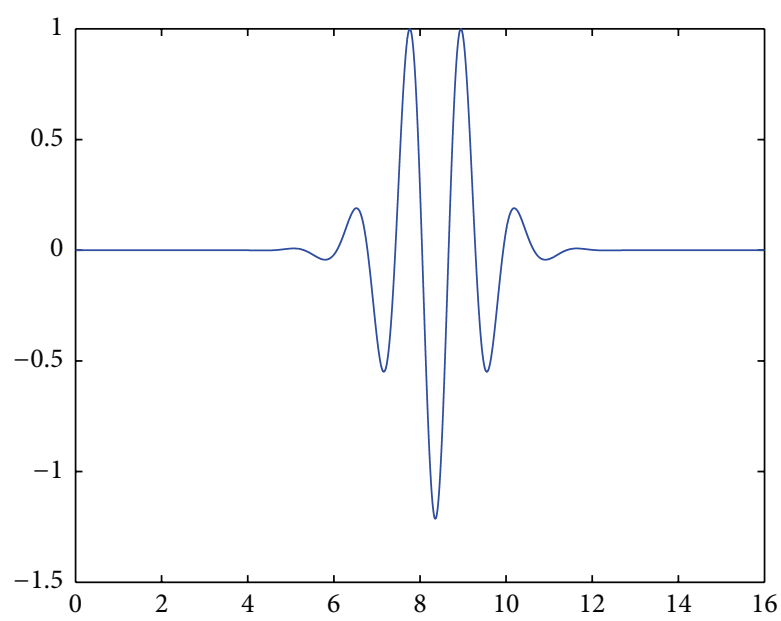

(b)

Figure 1: (a) The new refinable function with $\tau^{7,1,0.05}$ derived from the convergence of cascade algorithms and (b) the corresponding symmetric tight framelet, which provides approximation order of 4 .

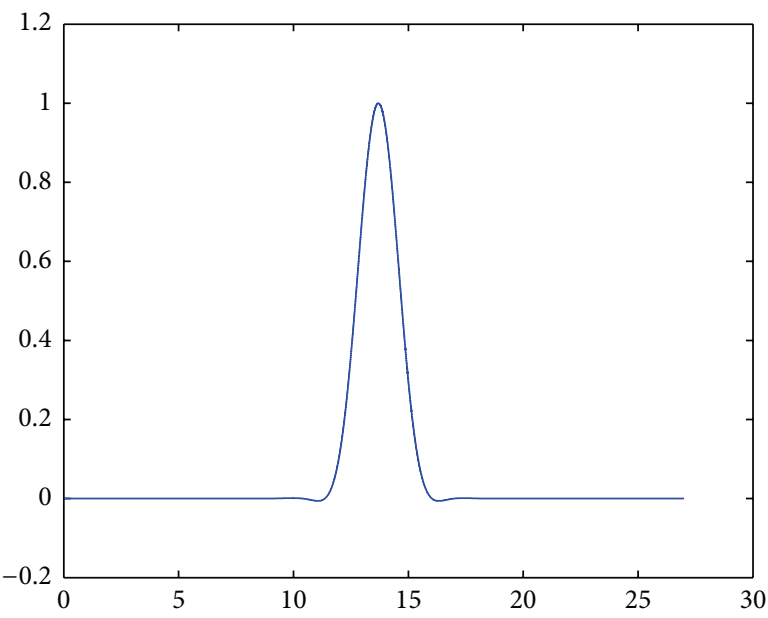

(a)

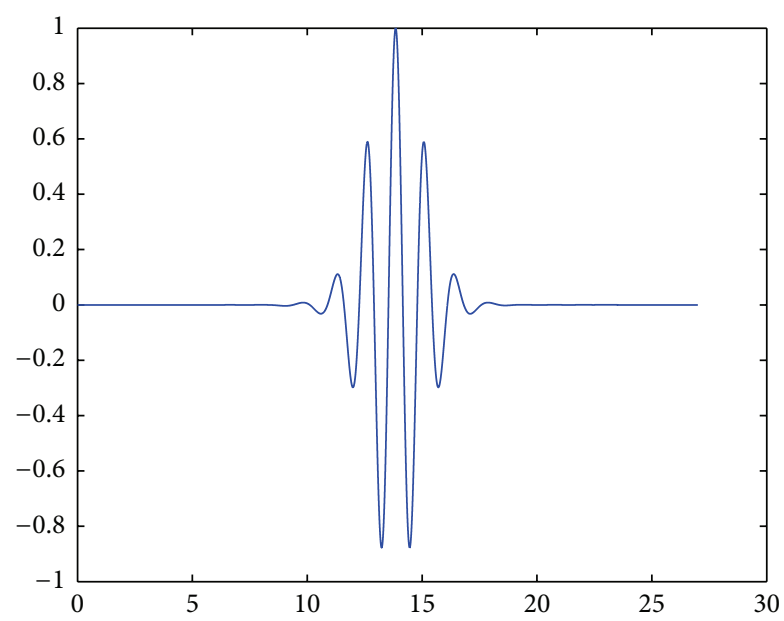

(b)

FIGURE 2: (a) The new refinable function with $\tau^{12,1,0.05}(\omega)$ derived from the convergence of cascade algorithms and (b) the corresponding symmetric tight framelet, which provides approximation order of 4.

convergence of cascade algorithms, symmetry, and approximation orders is analyzed.

\section{Conclusions}

In this paper, we study new marks (2) based on generalized Bernstein polynomials (1):

$$
\tau^{m, l, \alpha}(\omega):=\cos ^{2}\left(\frac{\omega}{2}\right)\left(\cos ^{2}\left(\frac{\omega}{4}\right)-\frac{\sum_{j=0}^{l}\left(\begin{array}{c}
m+l \\
j
\end{array}\right)\left(\prod_{k=0}^{j-1}\left(\cos ^{2}(\omega / 2)+k \alpha\right) \prod_{k=0}^{m+l-j-1}\left(\sin ^{2}(\omega / 2)+k \alpha\right)\right)}{\prod_{k=1}^{m+l-1}(1+k \alpha)}\right),
$$

with two positive integers $l, m$, satisfying $l<m-5$, to provide derived properties. The convergence of cascade algorithms in Theorem 9 is obtained, which guarantees the existence of refinable functions. In Theorem 11, we analyze the symmetry of the refinable functions, which is of importance. The regularity and approximation order of the new refinable functions are given; at the same time, the lower bound of the regularity exponents of refinable functions is showed by estimating the decay rates of their Fourier transform. Finally, we construct tight and sibling 
frames and demonstrate interorthogonality of sibling frames in Section 6. And, numerical examples are given to illustrate the construction of the proposed approach.

\section{Competing Interests}

The authors declare that they have no competing interests.

\section{Authors' Contributions}

All authors contributed equally in this paper. They read and approved the final paper.

\section{Acknowledgments}

This research is supported by the National Natural Science Foundation of China (no. 61271010) and Beijing Natural Science Foundation (no. 4152029).

\section{References}

[1] A. Ron and Z. Shen, "Affine systems in L2(Rd): the analysis of the analysis operator," Journal of Functional Analysis, vol. 148, no. 2, pp. 408-447, 1997.

[2] C. K. Chui and W. He, "Compactly supported tight frames associated with refinable functions," Applied and Computational Harmonic Analysis, vol. 8, no. 3, pp. 293-319, 2000.

[3] B. Han, "Symmetric tight framelet filter banks with three highpass filters," Applied and Computational Harmonic Analysis, vol. 37, no. 1, pp. 140-161, 2014.

[4] C. K. Chui, W. He, and J. Stöckler, "Compactly supported tight and sibling frames with maximum vanishing moments," Applied and Computational Harmonic Analysis, vol. 13, no. 3, pp. 224$262,2002$.

[5] I. Daubechies, B. Han, A. Ron, and Z. W. Shen, "Framelets: MRA-based constructions of wavelet frames," Applied and Computational Harmonic Analysis, vol. 14, no. 1, pp. 1-46, 2003.

[6] A. Z. Averbuch, V. A. Zheludev, and T. Cohen, "Tight and sibling frames originated from discrete splines," Signal Processing, vol. 86, no. 7, pp. 1632-1647, 2006.

[7] F. Abdelnour, "Symmetric wavelets dyadic sibling and dual frames," Signal Processing, vol. 92, no. 5, pp. 1216-1229, 2012.

[8] B. Dong and Z. Shen, "Pseudo-splines, wavelets and framelets," Applied and Computational Harmonic Analysis, vol. 22, no. 1, pp. 78-104, 2007.

[9] Z. Q. Shen and Z. Q. Xu, "On B-spline framelets derived from the unitary extension principle," SIAM Journal on Mathematical Analysis, vol. 45, no. 1, pp. 127-151, 2013.

[10] B. Han, "Compactly supported tight wavelet frames and orthonormal wavelets of exponential decay with a general dilation matrix," Journal of Computational and Applied Mathematics, vol. 155, no. 1, pp. 43-67, 2003.

[11] L. Z. Peng and H. H. Wang, "Construction for a class of smooth wavelet tight frames," Science in China Series F: Information Sciences, vol. 46, no. 6, pp. 445-458, 2003.

[12] A. Petukhov, "Symmetric framelets," Constructive Approximation, vol. 19, no. 2, pp. 309-328, 2003.

[13] B. Han and Q. Mo, "Tight wavelet frames generated by three symmetric $B$-spline functions with high vanishing moments,"
Proceedings of the American Mathematical Society, vol. 132, no. 1, pp. 77-86, 2004.

[14] B. Han, "The projection method for multidimensional framelet and wavelet analysis," Mathematical Modelling of Natural Phenomena, vol. 9, no. 5, pp. 83-110, 2014.

[15] B. Han, Q. Mo, and Z. Zhao, "Compactly supported tensor product complex tight framelets with directionality," SIAM Journal on Mathematical Analysis, vol. 47, no. 3, pp. 2464-2494, 2015.

[16] C. Bao, H. Ji, and Z. Shen, "Convergence analysis for iterative data-driven tight frame construction scheme," Applied and Computational Harmonic Analysis, vol. 38, no. 3, pp. 510-523, 2015.

[17] B. Han and Q. Mo, "Symmetric MRA tight wavelet frames with three generators and high vanishing moments," Applied and Computational Harmonic Analysis, vol. 18, no. 1, pp. 67-93, 2005.

[18] B. Han and Z. P. Zhao, "Tensor product complex tight framelets with increasing directionality," SIAM Journal on Imaging Sciences, vol. 7, no. 2, pp. 997-1034, 2014.

[19] B. Han and X. Zhuang, "Smooth affine shear tight frames with MRA structure," Applied and Computational Harmonic Analysis, vol. 39, no. 2, pp. 300-338, 2015.

[20] M.-J. Lai and J. Stöckler, "Construction of multivariate compactly supported tight wavelet frames," Applied and Computational Harmonic Analysis, vol. 21, no. 3, pp. 324-348, 2006.

[21] R. P. Boyer and L. C. Thiel, "Generalized Bernstein polynomials and symmetric functions," Advances in Applied Mathematics, vol. 28, no. 1, pp. 17-39, 2002.

[22] B. Han, "Refinable functions and cascade algorithms in weighted spaces with Hölder continuous masks," SIAM Journal on Mathematical Analysis, vol. 40, no. 1, pp. 70-102, 2008.

[23] I. Daubechies, Ten Lectures on Wavelets, vol. 61 of CBMS-NSF Regional Conference Series in Applied Mathematics, SIAM, 1992.

[24] Q. Jiang and Z. W. Shen, "On existence and weak stability of matrix refinable functions," Constructive Approximation, vol. 15, no. 3, pp. 337-353, 1999. 


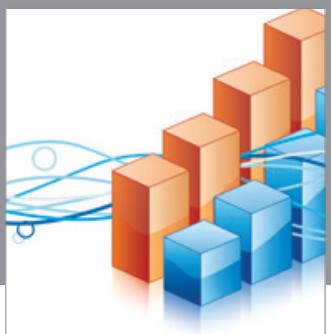

Advances in

Operations Research

vatem alat4

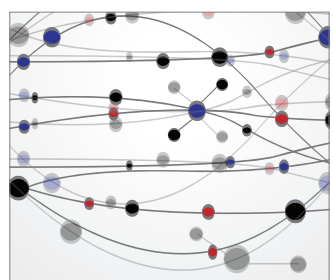

\section{The Scientific} World Journal
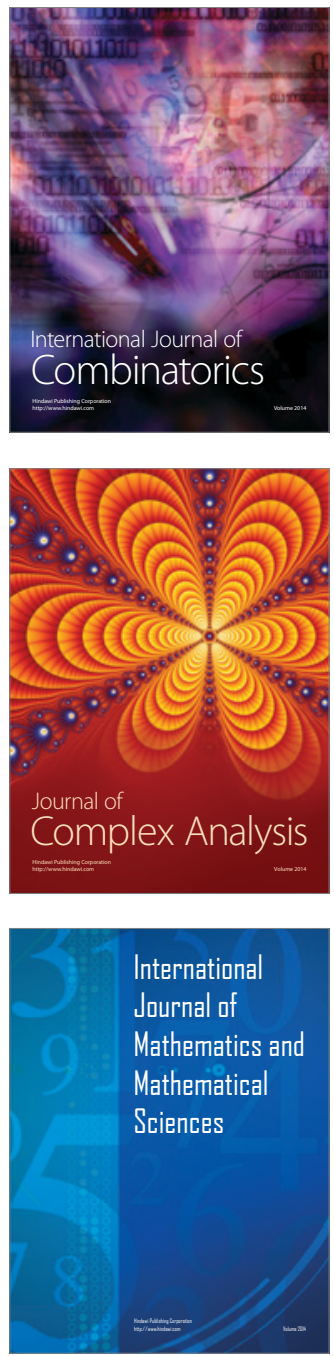
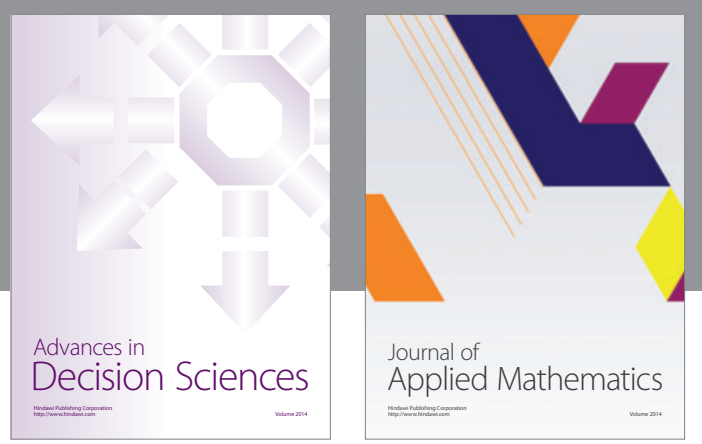

Algebra

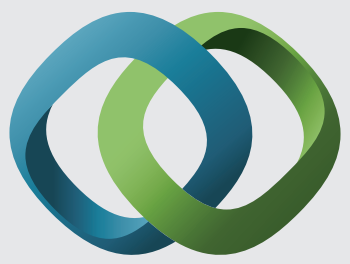

\section{Hindawi}

Submit your manuscripts at

http://www.hindawi.com
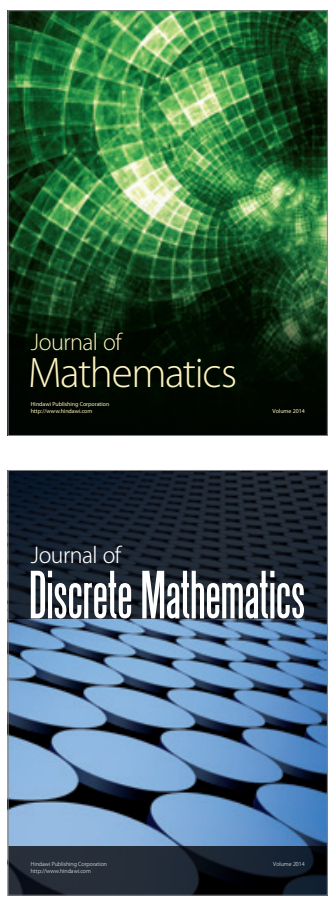

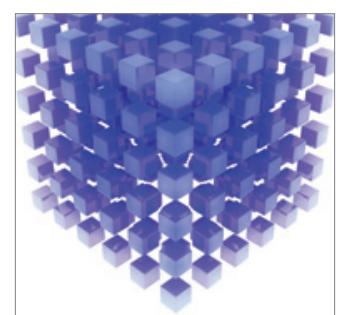

Mathematical Problems in Engineering
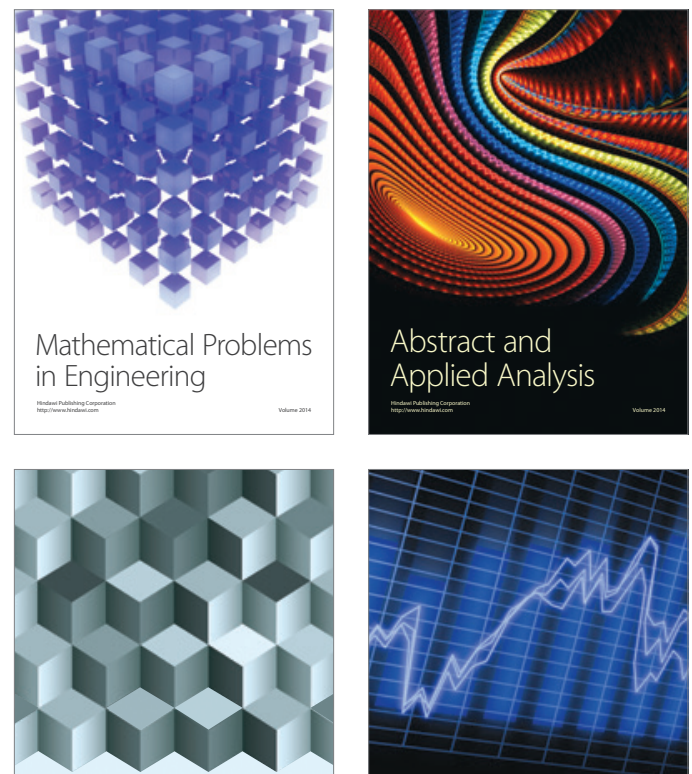

Journal of

Function Spaces

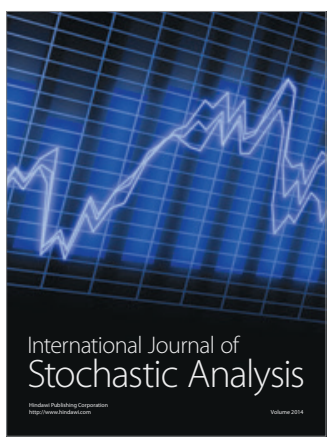

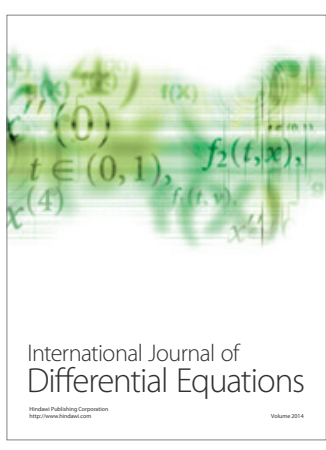
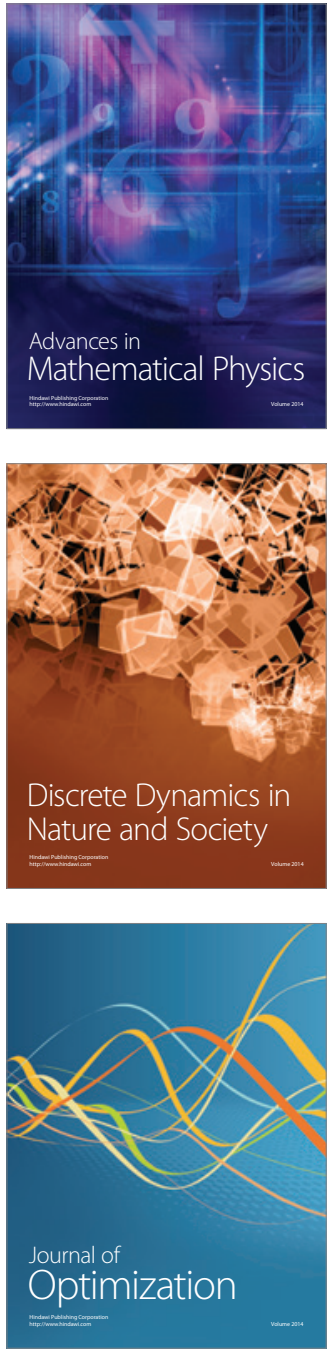